\title{
Optical Properties of Silver Nanoparticles for Surface Plasmon Resonance (SPR)-Based Biosensor Applications
}

\author{
Lufsyi Mahmudin ${ }^{1,2}$, Edi Suharyadi1* ${ }^{*}$, Agung Bambang Setio Utomo1, Kamsul Abraha1 \\ ${ }^{1}$ Department of Physics, Universitas Gadjah Mada, Yogyakarta, Indonesia \\ ${ }^{2}$ Department of Physics, Universitas Tadulako, Palu, Indonesia \\ Email: ${ }^{*}$ esuharyadi@ugm.ac.id
}

Received 1 June 2015; accepted 19 July 2015; published 22 July 2015

Copyright (C) 2015 by authors and Scientific Research Publishing Inc.

This work is licensed under the Creative Commons Attribution International License (CC BY). http://creativecommons.org/licenses/by/4.0/

(c) (i) Open Access

\begin{abstract}
It has been successfully carried out the synthesis of silver nanoparticles by chemical reduction method. Silver nitrate $\left(\mathrm{AgNO}_{3}\right)$ is used as the metal precursor and trisodium citrate as the reducing agent as well as the use of polyvinyl alcohol (PVA) as a stabilizer. The formation of silver nanoparticles was observed visually with discoloration (yellow). To excite surface plasmons, attenuated total reflection (ATR) method is used with Krestchmann configuration of the prism coupling. The maximum absorption band in the UV-Vis spectrometer shows a red shift of $429.43 \mathrm{~nm}$ wavelength for a colloidal solution of silver nanoparticles without PVA and $429.01 \mathrm{~nm}$ with PVA. The addition of PVA sharpened absorption spectrum curve and produce a broad absorption band which is indicative of a smaller particle size. TEM images showed that the morphology (crystallites) silver nanoparticles have nearly spherical geometry with dispersive particle distribution. Dispersibility of nanoparticles such as this could potentially be used as an active ingredient of SPR biosensor. The observation of the SPR phenomenon shows the SPR angle shift of $0.1^{\circ}$ when a thin layer of silver as an active ingredient a biosensor coated with silver nanoparticles and $0.2^{\circ}$ when silver nanoparticles with PVA. SPR angle shift and increase the reflectance values caused by changes in surface Plasmon, which can be a reference that the SPR phenomenon with the sensing surface modification using an additional layer of silver nanoparticles can increase sensitivity.
\end{abstract}

\section{Keywords}

Chemical Reduction, Silver Nanoparticles, Surface Plasmon

\footnotetext{
${ }^{*}$ Corresponding author.
}

How to cite this paper: Mahmudin, L., Suharyadi, E., Utomo, A.B.S. and Abraha, K. (2015) Optical Properties of Silver Nanoparticles for Surface Plasmon Resonance (SPR)-Based Biosensor Applications. Journal of Modern Physics, 6, $1071-1076$. http://dx.doi.org/10.4236/jmp.2015.68111 


\section{Introduction}

Biosensor based surface plasmon resonance (SPR) is a type of optical sensor that utilizes surface plasmon polariton (SPP) waves. Utilization of SPR as biosensors to monitor and provide information about biological processes shows very promising results especially in studying biomolecular interactions. SPR-based biosensor is very sensitive and responsive to changes in the refractive index of the analyte, so biosensors of this type can be used to detect the presence of biomolecules with biorecognition element which is used as the material of the analyte in the form of DNA [1] [2], protein [3] [4], enzymes [5], antibodies [6] [7] and peptides [8]. Meanwhile, in recent years, the noble metal nanoparticles have been the subject of research interest because of its unique properties, such as the electronic properties, optical, mechanical, magnetic and chemical properties are significantly different from the bulk material [9]-[11]. For this reason the noble metal nanoparticles have been widely used in many applications in different fields such as catalysis, electronic and photonic [12]-[14]. One application of noble metal nanoparticles gives you an idea SPR-based biosensor development with the addition of nanoscale structures are designed so that they can run a particular optical function. Based on the phenomenon that is able to manipulate the addition of nanoparticles SPR detection parameters, then the idea of increased SPR performance with the addition of nanoparticles [15] [16].

The addition of a metal nanoparticle structure allows for very strong coupling between the electron resonances with the light coming simultaneously in metallic nanostructures [17]. Such a mechanism would produce the amplified signal for its ability to propagate waves simultaneously. Research on added above a thin layer of metal nanoparticles has been carried out by Singh, et al. [18]. Singh, et al. (2009) reported that the presence of metal nanoparticles can shift ATR curve of $1.037^{\circ}$ compared with conventional SPR only shifts the ATR curve at $0.716^{\circ}$ in the experimental detection of BSA (Bovine Serum Albumin). Wu and Wang (2009) also developed a technique increased sensitivity sensing biomolecules using nanoparticles embedded in $\mathrm{SiO}_{2}$ matrix [19]. In addition, nanoparticles are selected for the ability to bind the material is very high considering that interacts with a surface area of the material is very wide compared to its bulk phase. With the ability of absorption material is very high, the mobility of the analyte can also be reduced, thus the accuracy and sensitivity of the biosensor will be improved.

In this study, SPR biosensor based on the conventional SPR models will be developed by adding silver nanoparticles structure on top of a thin layer. The addition of silver nanoparticles is expected to increase the sensitivity of SPR biosensor. In this method, the addition of colloidal silver nanoparticles affects the ATR curves, i.e. the shift of the SPR reflectance angle, extending the curve and an increase in the minimum reflectivity. On the other hand, various methods of preparation of silver nanoparticles have also been reported, such as electrochemical synthesis, ultrasonic irradiation, radiolysis, thermal decomposition and the simplest method is the chemical reduction of metal salts [20]-[23]. For this reason, this study proposes a new method in the synthesis of silver nanoparticles of silver nitrate solution with a reducing agent trisodium citrate and growth of its nanoparticles controlled using stabilizers polyvinyl alcohol. The size, structure and optical properties of the resulting nanoparticles subsequently were characterized by transmission electron microscope (TEM) and UV-Vis spectroscope.

\section{Experimental Methods}

\subsection{Experiment}

Silver nitrate $\left(\mathrm{AgNO}_{3}\right)$, trisodium citrate $\left(\mathrm{Na}_{3} \mathrm{C}_{6} \mathrm{H}_{5} \mathrm{O}_{7} \cdot 2 \mathrm{H}_{2} \mathrm{O}\right.$, Naci) and polyvinyl alcohol (PVA) was purchased from Sigma Aldrich. The colloidal silver nanoparticles (AgNPs) was prepared by using chemical reduction method. All solutions of reacting materials were prepared in aquadest. In typical experiment $100 \mathrm{ml}$ of $1.10^{-3} \mathrm{M}$ $\mathrm{AgNO}_{3}$ was heated with temperature of $90^{\circ} \mathrm{C}$ for 5 minutes. To this solution $10 \mathrm{ml}$ of $0.038 \mathrm{M}$ Naci was added drop by drop with constant stirring at $800 \mathrm{rpm}$ for 10 minutes until it changes color (pale yellow). The solution was heated continuously until the color changes were apparent (yellow). Then the heating is stopped and stirred continuously until cool at room temperature. Figure 1 (inset) shows colloidal silver nanoparticles resulting from the synthesis process. A1 shows colloidal silver nanoparticles were prepared by using Naci, while $\mathbf{A} 2$ with added stabilizers Polyvinyl Alcohol (PVA) with $\mathrm{AgNO}_{3}$ :PVA mass ratio of 1:10.

\subsection{Characterization}

The reduction of metal ions was monitored by measuring the UV-Vis spectroscopy of the solution. The UV-Vis 
spectroscopy measurement of silver nanoparticle was recorded on Shimadzu dual beam spectrophotometer (model UV-1650 PC) operated at a resolution of $1 \mathrm{~nm}$. The silver nanoparticle was measured in a wavelength ranging from 350 to $800 \mathrm{~nm}$. The TEM sample was prepared by placing one droplet of the prepared colloid onto a micro grid. A Hitachi H-800 model (voltage $200 \mathrm{kV}$ ) was employed for TEM observation.

\subsection{A Thin Layer Growth and Observations SPR}

Thin film layer of silver (Ag) on the prism surface was fabricated by a vacuum evaporator. As for the deposition of silver nanoparticles use the spray method. To excite surface plasmons, attenuated total reflection (ATR) method is used with Krestchmann configuration the prism coupling.

\section{Results and Discussion}

It has been successfully carried out the synthesis of silver nanoparticles by chemical reduction method. The formation of silver nanoparticles was observed visually with discoloration (yellowish). Yellowish color is formed on the sample indicates that the colloidal nanoparticles produced are the synthesis process is dominated by grains of silver nanoparticles.

UV-Vis spectrometer is spectral techniques are widely used to confirm the formation and structural characterization of silver nanoparticles in colloidal solution. Figure 1 shows the absorption spectrum of a solution of colloidal silver nanoparticles synthesized by Naci reducing agent and with different treatment. Optical absorption spectra of silver nanoparticles is dominated by surface plasmon which shows a shift towards the red end or blue end depends on particle size, shape, state of aggregation and the surrounding dielectric medium. Absorption band in the visible region is characteristic of silver nanoparticles. Plasmon peak and full width half maximum depends on the extent of aggregation of colloidal occur. Figure 1 shows the surface plasmon absorption band with a maximum of $429.43 \mathrm{~nm}$ for the spectrum of colloidal silver nanoparticles were prepared by reducing Naci. The addition of PVA stabilizers have sharpen absorption spectrum curves and occur redshift peak wavelength absorption at a wavelength of $429.01 \mathrm{~nm}$. The addition of PVA also delivers a broad absorption band which is indicative of a smaller particle size.

The absorption spectrum (Figure 1) of the pale yellow colloidal silver nanoparticles prepared by Naci reduction showed a surface plasmon absorption band with a maximum of $429.43 \mathrm{~nm}$ indicating the presence of spherical or roughly spherical Silver nanoparticles [24], and TEM imaging confirmed this (Figure 2). Morphological image in Figure 2 also show that the morphology (crystallites) silver nanoparticles have nearly spherical geometry with particle distribution is quite dispersive although there is a trend going on clotting. Dispersibility of

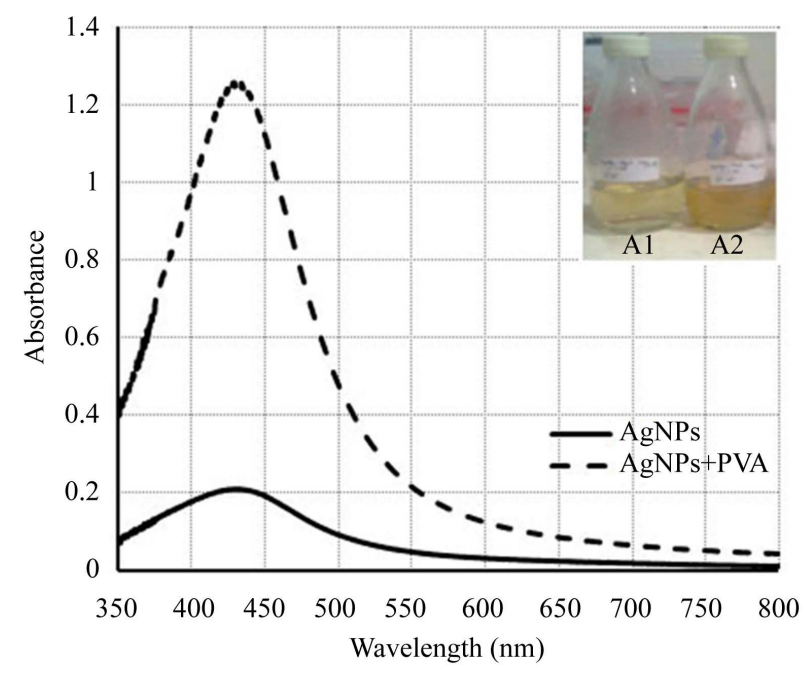

Figure 1. UV-Vis absorption spectra of colloidal silver nanoparticles synthesized and inserted photo synthesized silver nanoparticles. 


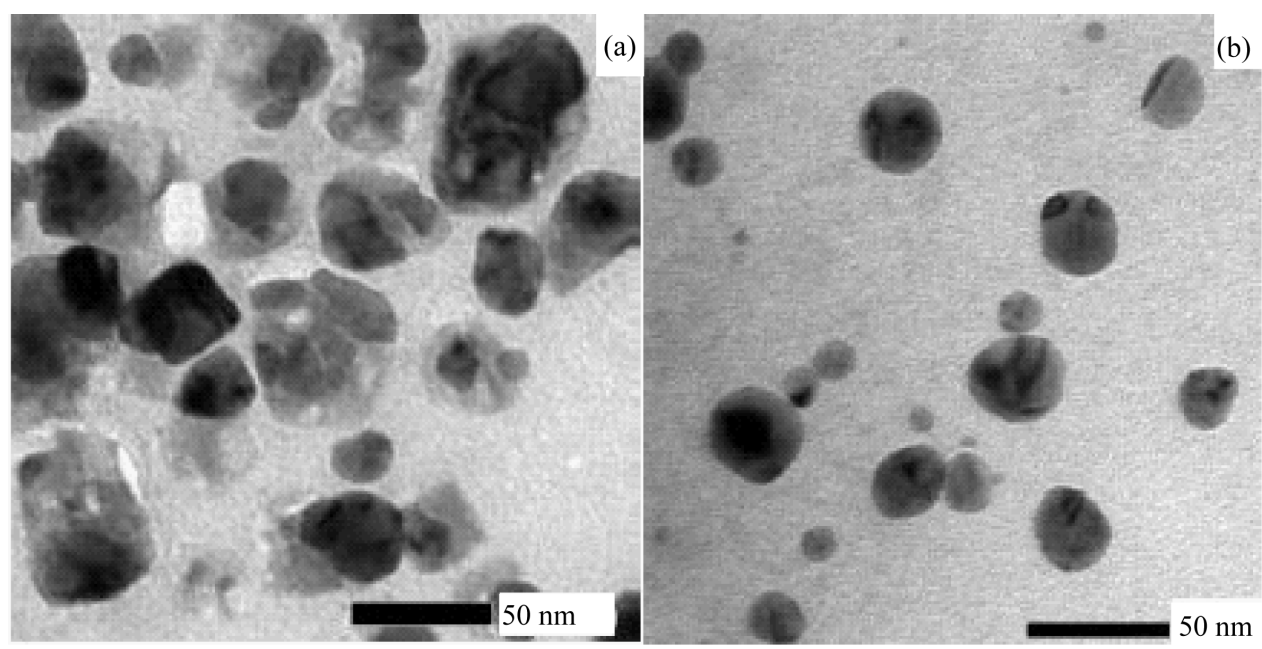

Figure 2. TEM image of colloidal silver nanoparticles with: (a) without stabilizers polyvinyl alcohol (PVA); and (b) with the stabilizer of PVA.

nanoparticles such as this could potentially be used as an active ingredient of SPR biosensor. TEM image also shows that the highest population size of the diameter of the two samples of silver nanoparticles in the range of 8 to $46 \mathrm{~nm}$ with an average diameter of the distribution obtained for $29 \mathrm{~nm}$ and $21 \mathrm{~nm}$.

Observations SPR phenomenon in this study using a prism/silver thin film (AgTF)/silver nanoparticles (AgNPs) system with Attennuated Total Reflection (ATR) methods in the Kretschmann configuration. Prisms are used in this research is the BK7 type prism with a refractive index of 1.51. Figure 3 shows the SPR reflectance curve for the prism/AgTF/AgNPs coating system. Silver thin film with the silver nanoparticles results a shift in the SPR angle, as shown in Figure 3. Prism/AgTF coating system has SPR angle of $42.80^{\circ}$ with a reflectance value of 0.445 and a surface plasmon wave number of $1.0113 \times 10^{7} \mathrm{~m}^{-1}$. After a silver thin film coated with silver nanoparticles, the SPR angle shift as far as $0.1^{\circ}$ to $42.90^{\circ}$ with reflectance values sharpened be 0.320 and the surface plasmon wave number into $1.0132 \times 10^{7} \mathrm{~m}^{-1}$. Surface plasmon waves number change caused by changes in the dielectric constant due to the addition of a layer of silver nanoparticles on the surface of a silver thin film.

Meanwhile, the use of stabilizers PVA in the synthesis of colloidal silver nanoparticles led to a shift in the SPR angle as far as $0.2^{\circ}$ becomes $43.00^{\circ}$ with reflectance values sharpened at 0.263 and surface plasmon wave number into $1.0151 \times 10^{7} \mathrm{~m}^{-1}$. Surface plasmon waves number change also caused by changes in the dielectric constant due to the mixing of the PVA polymer in a solution of colloidal silver nanoparticles. SPR angle shift and increase the reflectance values caused by changes in surface plasmon waves number caused by changes in dielectric constant. The larger the surface plasmon wave number, the greater the resulting SPR angle. The results of these observations may be a reference to that SPR phenomenon with the sensing surface modification using an additional layer of silver nanoparticles can increase sensitivity.

\section{Conclusion}

The synthesis of silver nanoparticles has been successfully carried out by chemical reduction method. The maximum absorption band in the UV-Vis spectrometer shows a red shift wavelength of $429.43 \mathrm{~nm}$ for a colloidal solution of silver nanoparticles without PVA and $429.01 \mathrm{~nm}$ with PVA. The addition of PVA sharpen absorption spectrum curve and produce a broad absorption band which is indicative of a smaller particle size. TEM images showed that the morphology (crystallites) silver nanoparticles have nearly spherical geometry with dispersive particle distribution. The observation of the SPR phenomenon shows the SPR angle shift of $0.1^{\circ}$ when a thin layer of silver as an active ingredient a biosensor coated with silver nanoparticles and $0.2^{\circ}$ when silver nanoparticles with PVA. SPR angle shifts and increases the reflectance values caused by changes in surface Plasmon, which can be a reference that the SPR phenomenon with the sensing surface modification using an additional layer of silver nanoparticles can increase sensitivity. 


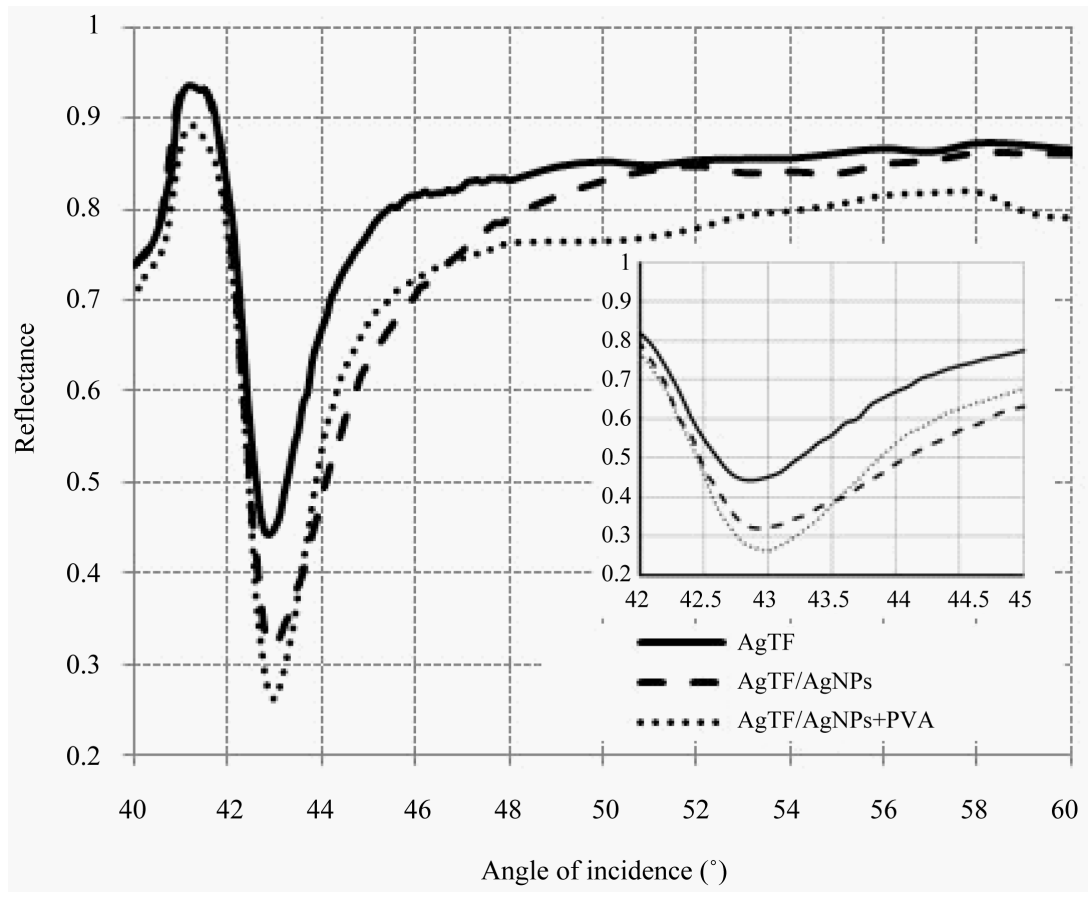

Figure 3. The reflectance curve in SPR observation.

\section{Acknowledgements}

This research is partly supported by Hibah Penelitian Fundamental (2015) and Hibah Kompetensi (2015), Ministry of Research, Technology and Higher Education, Republik Indonesia.

\section{References}

[1] Wang, Q., Yang, X. and Wang, K. (2007) Sensors and Actuators, 123, 227-232. http://dx.doi.org/10.1016/j.snb.2006.08.012

[2] Spadavecchia, J., Manera, M.G., Quaranta, F., Siciliano, P. and Rella, R. (2005) Biosensors and Bioelectronics, 21, 894-900. http://dx.doi.org/10.1016/j.bios.2005.02.016

[3] Pan, S., Xu, J., Shu, Y., Wang, F., Xia, W., Ding, Q., Xu, T., et al. (2010) Biosensors and Bioelectronics, 26, 850-853. http://dx.doi.org/10.1016/j.bios.2010.08.007

[4] Ritzefeld, M. and Sewald, N. (2012) Journal of Amino Acids, 2012, Article ID: 816032. http://dx.doi.org/10.1155/2012/816032

[5] Henn, C., Boettcher, S., Steinbach, A. and Hartmann, R.W. (2012) Analytical Biochemistry, 428, 28-30. http://dx.doi.org/10.1016/j.ab.2012.05.024

[6] Kausaite-minkstimiene, A., Ramanaviciene, A. and Ramanavicius, A. (2009) Analyst, 134, 2051-2057. http://dx.doi.org/10.1039/b907315a

[7] Buhl, A., Page, S., Heegaard, N.H.H., Von Landenberg, P. and Luppa, P.B. (2009) Biosensors and Bioelectronics, 25, 198-203. http://dx.doi.org/10.1016/j.bios.2009.06.037

[8] Ananthanawat, C., Vilaivan, T., Mekboonsonglarp, W. and Hoven, V.P. (2009) Biosensors and Bioelectronics, 24, 3544-3549. http://dx.doi.org/10.1016/j.bios.2009.05.011

[9] Evanoff Jr., D.D. and Chumanov, G. (2005) Small, 29634, 1221-1231. http://dx.doi.org/10.1002/cphc.200500113

[10] Kvítek, O., Siegel, J., Hnatowicz, V. and Švorčík, V. (2013) Journal of Nanomaterials, 2013, 1-15. http://dx.doi.org/10.1155/2013/743684

[11] Jain, P.K., Huang, X., El-Sayed, I.H. and El-Sayed, M.A. (2008) Accounts of Chemical Research, 41, 1578-1586.

[12] Yan, N., Xiao, C. and Kou, Y. (2010) Coordination Chemistry Reviews, 254, 1179-1218. http://dx.doi.org/10.1016/j.ccr.2010.02.015

[13] Yougen, H., Tao, Z., Pengli, Z. and Rong, S. (2012) Colloid and Polymer Science, 290, 401-409. 
http://dx.doi.org/10.1007/s00396-011-2555-0

[14] Chen, Y. and Ming, H. (2012) Review Literature and Arts of the Americas, 2, 37-49.

[15] Agata, R.D., Corradini, R., Ferretti, C., Zanoli, L., Gatti, M., Marchelli, R. and Spoto, G. (2010) Biosensors and Bioelectronics, 25, 2095-2100. http://dx.doi.org/10.1016/j.bios.2010.02.008

[16] Jiang, G.Q., Baba, A., Ikarashi, H., Xu, R.S., Locklin, J., Kashif, K.R., et al. (2007) The Journal of Physical Chemistry C, 111, 18687-18694. http://dx.doi.org/10.1021/jp075986e

[17] Berglind, E., Thylen, L. and Liu, L. (2010) IET Optoelectronics, 4, 1-16. http://dx.doi.org/10.1049/iet-opt.2008.0045

[18] Singh, K.N., Alqudami, A., Subaramanian, A., Sharma, V. and Muralidhar, K. (2009) AIP Conference Proceedings, 1147, 331-338. http://dx.doi.org/10.1063/1.3183453

[19] Wu, B. and Wang, Q.K. (2009) Optica Applicata, 39, 31-41.

[20] Pyatenko, A., Yamaguchi, M. and Suzuki, M. (2007) The Journal of Physical Chemistry C, 111, 7910-7917. http://dx.doi.org/10.1021/jp071080x

[21] Ghodselahi, T., Neishaboory, T. and Vesaghi, M.A. (2011) Biosensors, 2, 59-61.

[22] Tran, Q.H., Nguyen, V.Q. and Le, A. (2013) Advances in Natural Sciences: Nanoscience and Nanotechnology, 4, 1-20. http://dx.doi.org/10.1088/2043-6262/4/3/033001

[23] El-Nour, K.M.M.A., Al-Warthan, A. and Ammar, R.A.A. (2010) Arabian Journal of Chemistry, 3, 135-140. http://dx.doi.org/10.1016/j.arabjc.2010.04.008

[24] Guzmán, M.G., Dille, J. and Godet, S. (2008) World Academy of Science, Engineering and Technology, 43, 357-364. 